\title{
Functional Foods as the Prospective Therapeutic Option in Chronic Diseases. A Systemic Review
}

Anum Nazir ${ }^{1,2}{ }^{*}$, Nizwa Itrat ${ }^{1,2}$, Muhammad Awais Saleem ${ }^{2}$, Mahr Un Nisa ${ }^{2}$, Aleena Shahid ${ }^{1}$, Zain Mushtaq ${ }^{3,4}$, Hafiz Abdul Rafey ${ }^{5}$

${ }^{1}$ Department of Nutrition and Dietetics, the University of Faisalabad Punjab, Pakistan.

${ }^{2}$ Departments of Nutritional Sciences, Government College University Faisalabad Punjab, Pakistan.

${ }^{3}$ Institute of Soil and Environmental Sciences, University of Agriculture, Faisalabad, Pakistan.

${ }^{4}$ Department of Soil Science, University of the Punjab, Lahore, Pakistan

${ }^{5}$ Imran Idrees college of pharmacy Sialkot, Pakistan.

\section{ART ICLE INFO}

\section{Article History}

Received: August 21, 2021

Revised: October 24, 2021

Accepted: November 15, 2021

\section{Keywords}

Chronic Diseases

Functional Foods

Bioactive Compounds

Wellness

Sustain Healthy Lifestyle

\section{A B S T R A C T}

Chronic diseases become the leading cause of more than $50 \%$ of deaths around the world. The real reason is inflammation behind all chronic diseases like chronic kidney disease, ischemic heart disease, diabetes, and hypertension. There are several functional foods which involve in the management and treatment of many diseases because they provide nutrition and potential health benefits. Bioactive compounds in functional foods are responsible for all these health benefits present in them. The nutritional, physiological, and immunological properties of Nutraceutical foods promise health-promoting as well as disease-preventing potential. Natural food commodities such as whole-grain cereals, fruits and vegetables, nuts, healthy fats, low-fat dairy products, and some other nutraceutical compounds such as herbs and spices provide health benefits by promoting wellness, protecting against the diseases and their complications, slowing, or omitting the disease progression, promote healthy gut health, prevent from deficiencies, and help in to sustain the healthy lifestyle. The purpose of this systemic review was to describe the effects and outline some promising potentials of functional foods against disease prevention and treatment.

Corresponding Author: Anum Nazir

Email: anum.nazir@tuf.edu.pk

(c) The Author(s) 2021.

\section{INTRODUCTION}

With time, the diseases related to metabolic syndrome and immune system compromise becoming more vibrant and widely predominant serious health concern around the world. Several diseases lie under such as diabetes, obesity, hypertension, CVDs, and stroke. Increased oxidative stress and pro-inflammatory cytokines eventually lead to poor health. The prevalence of metabolic syndrome in Pakistan ranges from 18\% to $46 \%$ and is increasing day by day. Several factors are involved in developing the disease such as poor lifestyle, and eating habits, poor nutrition, sedentary lifestyle, social and behavior attitudes, age, and genetics. Unchanged lifestyle and dietary habits lead to multiple complications among patients suffering from chronic diseases. The classical idea of avoiding deficiencies and ensuring nutritional adequacy is important for the optimal nutritional status of patients. Nutraceutical foods are used as an alternative treatment therapy for the management of illnesses (Adam et al., 2003).

Functional foods involve in preventing disease and 
promote overall wellness. Functional foods provide the essential nutrients and bioactive compounds that play important role in the health benefits, prevent diseases and promote well-being. Numerous bioactive compounds include polyphenols, flavonoids, alkaloids, carotenoids, healthy fats include omega- 3 fatty acids and PUFA and enzymes. Functional foods include the protection against metabolic diseases by reducing oxidative stress and enhancing the anti-inflammatory cytokines in the body. Functional foods involved in the protection against diseases by enhancing the antioxidant activity in body (Adefegha, 2018).

Functional foods and their effects on cardiovascular diseases (CVD)

Heart disease is one of the leading causes of death throughout the world and cause more than $80 \%$ of mortality in the country which have a less and middle economy. According to the estimation of WHO 17.9 million people died from CVDs in 2019, which depicts 32 $\%$ of worldwide deaths. Heart attacks and stroke accounted for 85 percent of all deaths, with low- and middle-income nations accounting for three-quarters of CVD deaths. (World Health Organization, June 2021). Coronary heart disease, cerebrovascular disease, peripheral arterial disease, rheumatic heart disease (damage to the heart muscle and valves caused by streptococcal bacteria in rheumatic fever), congenital heart disease (birth defects caused by malformations of the heart structure from birth), and deep vein thrombosis and pulmonary embolism (blood clots in the lungs) are just a few of the CVD complications.

Associated risk factors of these all complications are Unhealthy eating habits, physical inactivity, usage of tobacco and harmful usage of alcohol. In 200816.3 million citizens were died is just due to CVDs, this was an estimated figure that was given by World Health Organization. (National Health Statistics Reports, 2009). Several functional meals have been demonstrated to help prevent and treat cardiovascular disease, which is the leading cause of mortality in the United States. These foods include oats, psyllium, flaxseed, tea, salmon, grapes, almonds, and stanol, as well as a sterol (Aguirre et al., 2008). When consumed in large quantities regularly, these foods may reduce the risk of cardiovascular disease by considerably decreasing the lipid profile, optimizing arterial conformity, minimizing plaque formation, scavenging free radicals, and avoiding platelet aggregation.

\section{Eicosatetraenoic acid (EPA) and Docosahexaenoic acid (DHA)}

Consumption of fish or fish oil has been shown to have anti-inflammatory qualities (Alipour et al., 2014). Longchain fatty acids (eicosatetraenoic acid [EPA] and docosahexaenoic acid [DHA]) found solely in seafood interact with arachidonic acid in cell membranes, affecting metabolic pathways that strengthen immune systems and reduce inflammation. Regular fish oil consumption has anti-inflammatory effects similar to those seen in low-arachidonic acid diets (i.e., vegan diets), and fish oil consumption actually amplifies the advantages of low-arachidonic acid diets (Ameye et al., 2006).

Fish oil has antithrombotic, triglyceride-lowering, and antiarrhythmic qualities, as well as anti-inflammatory properties, in CVD patients (Ameye et al., 2006). Furthermore, fish and fish oil consumption has been linked to a lower incidence of cardiovascular events, which could be due to the possible effects of EPA and DHA on plaque development and endurance (Anderson and Moore, 2004). For a median of 6 weeks, 192 patients undergoing carotid endarterectomy were randomly assigned to fish oil, sunflower oil, or control groups to measure plaque stability (Andrea, 2021). In patients who took fish oil, EPA and DHA were easily incorporated into the carotid plaque, and their plaques had less inflammation and a thin fibrous layer.

\section{Vitamin C, Carotenoids, Polyphenols, Potassium}

Overall inflammation and immune system activation are influenced by oxidative stress. People who eat a fruit and vegetable-rich diet have higher tissue levels of antioxidants including vitamin $\mathrm{C}$, carotenoids, and polyphenols, as well as a decreased risk of heart disease (Ankola et al., 2007; Asher et al., 2006). In controlled clinical trials, vitamin C lowered CRP concentrations by 20-30 \% (Cencic and Chingwaru, 2010), and this vitamin $\mathrm{C}$-induced decrease in CRP was associated to therapeutic advantages in cardiac patients. Vitamin $\mathrm{C}$ is abundant in citrus fruits, red and yellow peppers, Brussels sprouts, melons, and strawberries. Vitamin C's high antioxidant capabilities, both extracellular and intracellular, contribute to tissue ROS reduction (Block et al., 2009). Carotenoids, such as lycopene and beta-carotene, which can be found in colorful foods like carrots, tomatoes, and dark green vegetables, are another type of dietary antioxidant that can help reduce oxidative stress in vivo as well as blood markers of inflammation. Tomato juice 
was added to typical people's diets (two cups per day for two weeks) and resulted in significantly lower plasma levels of CRP (-17 \%) and TNF- (-44\%) [15]. Individuals with grade 2 diabetes had considerably lower levels of LDL cholesterol oxidation in plasma, as well as systolic and diastolic blood pressure measures (Boldo et al., 2010).

Vitamin C, vitamin E, betacarotene, and other related carotenoids, as well as the minerals selenium and manganese, are among the hundreds, if not thousands, of distinct chemicals that can act as antioxidants found in many fruits and vegetables. Flavonoids, phenols, polyphenols, phytoestrogens, and a slew of other compounds are among them. Dairy products containing probiotics, such as yogurt and cheese, as well as milk containing omega-3 fatty acids, play an important role in the creation of functional meals.

Patients with cardiovascular disease who had fasted overnight were given either red grape extract or a placebo, and postprandial endothelial cell activity was assessed over two hours by measuring flow-mediated brachial artery widening (Bolton-Smith et al., 1992). Red grape extract improved flow-mediated elongation by 65 percent as compared to the placebo. In healthy adults, raisins (120 $\mathrm{mL}$ per day for 42 days) substantially decreased TNF- and intracellular adhesion molecules (Cortés et al., 2006; Chaturvedi, 2012). Tea, coffee, and chocolate, for instance, are abundant in polyphenols and have been found to reduce oxidative stress markers in controlled feeding studies. Since growing research shows an inverse connection between blood pressure and dietary intake of fruits and vegetables, potassium might just have a potential preventative function in the treatment of cardiovascular disease (Jenkins et al., 2002; Engelhard et al., 2006).

\section{Monounsaturated and Polyunsaturated Fatty Acids (MUFA and PUFA)}

Numerous large prospective cohort studies have demonstrated that nuts, flaxseeds, and peanuts reduce cardiovascular disease risk, and replacing a serving of nuts in the usual diet with equal energy from carbs or saturated fat reduces cardiovascular events by around 30 \% (Fraser, 1999; Fritsche, 2006). Nuts are high in monounsaturated and polyunsaturated fatty acids, arginine (a precursor to the vasodilator nitric oxide), dietary fiber, and a range of antioxidant polyphenols, which all help to lower cholesterol. A single serving of walnuts (three oz) as part of a high saturated fat meal (salami and cheese sandwich with high-fat yogurt) reduced postprandial vascular reactivity, an emerging side effect for heart diseases, in individuals with hypercholesterolemia in a controlled trial using olive oil as the control group. Postprandial vascular reactivity is defined by decreased nitric oxide bioavailability and increased production of pro-inflammatory cytokines and cellular adhesion molecules. Hyper-cholesterolemic individuals who ate walnuts regularly ( 7 to 14 nuts daily for a month) had better endothelium function than those who followed a Mediterranean-style diet for a longer period (Gila-Diaz, 2021).

\section{Iso-flavonoids}

Various Isoflavonoids (genistein, daidzein, and glycitin) present in soy products are organic phytoestrogens that reduce the risk of atherosclerosis by preventing LDL oxidation (Abete et al., 2008). Numerous studies have found that eating soy protein decreases the oxidation susceptibility of LDL particles (Gu et al., 2014). Furthermore, isoflavone-rich soy protein decreased the oxidation vulnerability of LDL particles in healthy people (Guilleminault et al., 2017). Soy isoflavones have been found in meta-analyses of randomized clinical trials to reduce total and LDL cholesterol in people (Wiseman et al., 2000). Clinical studies on the effect of soy diets and isoflavone supplementation on blood lipids is less apparent. These conflicting findings might be explained by hypercholesterolemic patients having worse reactions than their control counterparts. Current/Recent epidemiological studies employing soy protein in postmenopausal women have found similar variations. There is clearly a need for more study to discover if there are differences between entire foods, soy protein, and isoflavone extracts (Heber, 2004).

\section{Vitamin E}

Vitamin E is a strong anti-inflammatory agent, especially at high doses, in addition to its action as a free radical scavenger (Houston, 2013). A rising body of evidence shows the existence of a strong negative connection between plasma vitamin $\mathrm{E}$ and CVD risk, as well as a link between vitamin E intake and CHD risk (Houston, 2013; Houston, 2014). Despite this, clinical studies have found no evidence that vitamin $\mathrm{E}$ can help prevent cardiovascular disease (Hu and Stampfer, 1999). After that, they all came to the same unfavorable conclusion after more than 90 investigations, meta-analyses, and systematic reviews. High-dose vitamin E (400 IU per day) was connected to an increased risk of overall mortality in 
a recent dosages meta-analysis. Because excessive vitamin $\mathrm{E}$ intake can induce blood thinning and lead to deadly hemorrhage, these are all undesirable consequences of using the wrong kind/or amount of vitamin E above the body's requirement. Blood clotting, which is our body's natural defense against excessive bleeding after an injury, can also be hampered. When used in sufficient amounts, it has also been associated to an increased risk of hemorrhagic stroke, or a strokeinduced by bleeding in the brain, which is required for successful oxidative stress mitigation. The contrast in results across observational and interventional trials suggests that tocopherol's preventative advantages occur in the context of other nutrients and that it is, therefore, most effective and helpful when consumed with meals (Huang et al., 2011).

\section{Functional foods effect on hypertension and stroke Vitamin D}

Vitamin D3 affects blood pressure and stroke in distinct ways (He et al., 1995; Jacob et al., 2008). Vitamin D may play an independent and direct function in blood pressure, insulin metabolism, and glucose control (Kafeshani et al., 2017). Vitamin D decreases vascular smooth muscle hypertrophy by suppressing proinflammatory cytokines, increasing nitric oxide (NO), improving endothelial function and arterial flexibility, and suppressing pro-inflammatory cytokines (Keller, 2011).

\section{Lycopene and Coenzyme Q10}

Lycopene intake lowers blood pressure, serum lipids, and oxidative stress indicators significantly (Khan et al., 2013). Vitamin C is abundant in grapefruit, watermelon, tomatoes, guava, pink apricots, and papaya. In hypertensive patients, coenzyme Q10 (ubiquinone) provides long-lasting and substantial antihypertensive effects (Korantzopoulos et al., 2005). CoQ10 enhances endothelial function and vascular flexibility by increasing eNOS and NO (Lee et al., 2016).

\section{Alpha-Lipoic Acid and Flavonoids}

Alpha-lipoic acid (ALA) is a well-known and potent antioxidant that occurs naturally in our diets, but when taken as a supplement in the form of a natural or synthetic isolate, it appears to have increased functional capability. The use of alpha-lipoic acid (ALA) in the treatment of hypertension and stroke, particularly in the context of metabolic syndrome is a hot topic in the scientific community right now. Lipoic acid reduces blood pressure by lowering oxidative stress, inflammation, and serum aldehydes, resulting in vasodilation, improved endothelial function, and lower blood pressure (Liu et al., 2000). Flavonoids (flavonols, flavones, and isoflavones) are potent free radical scavengers that have been shown to help prevent atherosclerosis, enhance arterial calm, and lower blood pressure.

\section{Functional foods effect on obesity}

Global epidemic obesity is directly associated with decreased life expectancy by increasing the risk for other diseases such as CVDs, hypertension, cancer, and diabetes (Matsunaga et al., 2014). Many factors contribute to the obesity that also faced by developed countries such as, high intake of energy foods, aging, sedentary lifestyle, and unbalanced nutrition (Medjakovic and Jungbauer, 2013). Obesity results from a lack of energy balance in the body. Obesity is continuing as a major public health concern (Mikhin et al., 2011).

Reducing body weight by increasing energy expenditure has positive influences on obesity. By uncoupling the proteins at mitochondria, the brown adipocyte tissues regulate heat production by excessive energy. Some obesity-related nutraceuticals that involve in thermogenesis used as anti-obesity. Green tea that has a powerful antioxidant known as Epigallocatechin gallate, that involved in the anti-obesity action by inducing thermogenesis and boosting the burn fat process. Caffeine is a natural stimulant present in coffee and caffeinated teas. Act as a well-known anti-obesity effect by promoting the fat oxidation, enhancing thermogenesis process in the body and boosting BMR. Capsaicin chemical compound present in spicy foods naturally belongs to peppers, jalapenos, and cayenne. This chemical compound boosts the caloric burn naturally and effectively impacts the appetite (Miller et al., 2008; Mirmiran et al., 2014). Cocoa powder involves in the anti-obesity effect shown by studies. The function includes in reduction of body weight naturally by improving insulin function in the body, reducing inflammation, increase fecal fat content (Morcos et al., 2001).

\section{Carbohydrates based food}

The content of fiber and glycemic index of carbohydrates are involved in obesity. High glycemic foods and low fiber intake lead to increase visceral fat and increased in weight. 


\section{Protein-based food}

Plays important role in metabolism due to the phenolic content of legumes such as phenolic acids, lignans and flavonoids (Mozaffarian, 2008). Several studies showed that the diet rich in proteins have many weights management benefits such as: Protein based food plays important role in metabolism due to the phenolic content of legumes such as phenolic acids, lignans and flavonoids (Mozaffarian, 2008). Several studies showed that the diet rich in proteins have many weights management benefits such as:

- Induce high satiety

- Induce thermogenic effect

- Suppress the appetite

- Increased the energy expenditure

\section{Fat based foods}

Selection of fat is necessary in weight management.

- Conjugated linoleic acid present in milk and meat and their products reduce the body fat accumulation and increase protein content (Nestel et al., 2015).

- Consumption of fatty fish increase leptin level (Ogden et al., 2014).

- Polyphenols rich oil reduce the inflammation (Ogden et al., 2014).

\section{Fruits and vegetables}

Because of their high bioactive chemical content, fruits and vegetables promote a wide range of health advantages. They include a variety of bioactive chemicals that have a vital function in the prevention of chronic illnesses. Anthocyanins rich fruits and vegetables blackcurrants, blackberries and blueberries, cabbage, cranberries, and cherries. help in reduction of weight gain and improve the serum glucose level (Okarter and Liu, 2010). Apples and pomegranate help in the lowering the plasma lipid levels, improve oxidation stress in body, reduce the inflammation and improve the insulin function in body to maintain the serum glucose level in blood (Paran et al., 2009). Bitter gourd plays the vital in role in the reduction of body weight by improve glucose and lipid level in body, promotes low protein oxidation reduce the inflammatory markers in body (Perez-Herrera et al., 2012). Onion peal tea act as natural anti-obesity drink by reduce the serum glucose and leptin levels in body, improve lipid profile and suppressed the increase in body weight (Prior et al., 2010).

Potential of nutraceuticals/ functional foods in managing the obesity by the involvement as the nutritional, physiological and immunological function in the GI tract

Functional / nutraceutical foods have been demonstrated to be possible dietary components that give health advantages in addition to appropriate nutritional impacts. They contribute to an enhanced condition of health and well-being. The functional foods/nutraceutical foods contribute to the proper function of the GI tract through several mechanisms of action that directly contribute to weight management and obesity prevention.

A variety of activity activities by functional foods are involved in the management and treatment of obesity by decreasing oxidative stress, inflammation, improving nutrition metabolism, and regulating appetite and hunger hormones. Functional foods, as part of a well-balanced diet, may aid in weight control and minimize the metabolic effects of obesity.

\section{Functional foods/ Nutraceutical compounds}

Increased antioxidant activity

Improve carbohydrates and lipid metabolism

Reduce inflammation

Improve the appetite and hunger hormones levels Weight management

\section{Functional foods and effect on asthma}

Asthma has become the worldwide public health concern (Puglisi et al., 2008). Defined as the allergic illness of complex gene-environment contact. Tendency is explained by the genetic factors. Over the decade the prevalence is increased by the involvement of many environmental factors that play dominant role in asthma etiology (Rahman et al., 2012). Lung's airway become inflamed, narrow, and swollen and increased mucus formation. Breathing process become difficult due to muscle tighter. Due to this patient encounters SOB, coughing, chest tightness and wheezing. The wellbalanced diet plays vital/important role in the cure and managing the asthma condition. Increase the fruits, vegetables, whole grain cereals and avoid saturated fat, processed food and reduced animal products leads towards the reduction of inflammatory makers, free radical in body, help in managing the asthma signs severity, Functional foods consumption involve in reliving the asthma symptoms. Eating well-rounded diet including functional foods improve the symptomatic condition of asthma (Table 1). 


\section{Diet and systemic inflammation}

Intake of vegetables and fruits as compared to western diet associated with worsen disease outcomes include increased airway inflammation, cause poor ling function, and increased the exacerbations these all are contributed to worsen the asthma condition. Impact of fruits and vegetables include:

Decrease proinflammatory cytokines.

Lower airway inflammation.

Produce immunomodulatory effect (Rayalam et al., 2008).

\section{Role of lipids}

Consumption of healthy fats and limited the intake of saturated fat have positive influences on asthma condition. Studies revealed that the polyunsaturated fatty acids have great influences on in the management on asthma. Polyunsaturated fatty acids involve in relieving the asthma condition by regulate the immune response and anti-inflammatory action in body (Reid et al., 2009).

\section{Role of antioxidant in asthma management}

Studies shown that Vitamin C, E, zinc, selenium, magnesium, flavonoids, carotenoids have great impact on asthma. These are involved in the prevention or reduction of inflammatory response in airways. Flavonoids have potent anti-allergic impact. Intake of a variety of nutrients in daily diets, relive the symptoms and lower the rate of asthma by different mechanism of action in the asthma prognosis Table 2 (Riegsecker et al., 2013).

Table 1. Role of nutrients.

\begin{tabular}{ll}
\hline Nutrients & Impact \\
\hline Sodium & Increased smooth muscle contraction \\
Magnesium & Relaxation of smooth muscle cells \\
Zinc and copper & Cofactor of superoxide dismutase \\
Selenium & Cofactor in glutathione, peroxidase \\
\hline
\end{tabular}

Table 2. Role of functional foods in relieving the asthma condition.

\begin{tabular}{ll}
\hline Functional foods & Impact \\
\hline Apples & Improve lung function by presence of phytochemicals \\
Banana & Due to high potassium content reduce wheezing symptoms \\
Omega 3 fatty acids & Reduce inflammation \\
\hline
\end{tabular}

\section{Functional foods and effects on arthritis}

Defined as the inflammation of joints. There are several conditions that effect the joints. More common include osteoarthritis, rheumatoid arthritis, and gout. Mostly the arthritis effects the aged above 65 or effect all children at any age. Commonly arthritis warning sign include pain, stiffness, inflammation, moving difficulties, swelling and fever. Possible causes include injury to joints, degenerative process, abnormal metabolism, immune dysfunction and inheritance. A lifestyle modification, and dietary changes is the leading way of arthritis prevention. Aim in treatment of arthritis is to minimize the pain and joint damage and improve the quality of life (Rissanen $e t$ al., 2003). Treatment through the healthy diet including functional foods is the better rather than the pharmacological interventions to provide the long-term benefits rather than the short-term. Medications used for longer time associated with the adverse health effects nevertheless, the functional foods and nutraceuticals provide the wide range of health benefits along with the devoid of worsen health effects (Ros et al., 2004). To healthy and balanced diet along with the use of functional foods help in the improve symptoms and slow the progression. Functional foods that are rich in antioxidant especially from fruits (apple, pomegranate, citrus fruit) and vegetables (vit-k rich- green vegetables) and herbs and spices include garlic, ginger, black pepper, turmeric etc. help in arthritis management by reduce inflammation, manage the severity of symptoms, and slow the progression of disease (Sackesen et al., 2008). Green tea: Polyphenols present in green tea is EGCG that act as the anti-inflammatory agent. Reduce the inflammatory markers and protects the joints from destruction (Salinthone et al., 2011).

Probiotics: The imbalance between the pathogenic and gut flora may lead to worsen the condition. Intake of probiotics (Cheese, yogurt, and pickles) helps in the management of arthritis by decrease the join pain, reduction in swelling and reduce the inflammatory markers in body (Scribner et al., 2008). 
Fish: Fish is the most abundant source of omega-3 fatty acids, which function as anti-inflammatory indicators. Tuna, salmon, and sardines should be included in the diet 2 to 3 times each week. C-reactive proteins and interleukin-6 are two inflammatory proteins that omega3 fatty acids decrease. Fish and fish oil supplements aid in the treatment of arthritis. Fish and fish oil supplements can assist with joint pain and stiffness, as well as decrease inflammation and relieve morning stiffness (Shaikh, 2008).

Olive oil and canola oil: These oils have antiinflammatory action by the presence of oleocanthal that helps in the reduction of inflammation. These two oils have the balance of omega 3 and omega 6 fatty acids. 2 to 4 tablespoon daily is recommended.

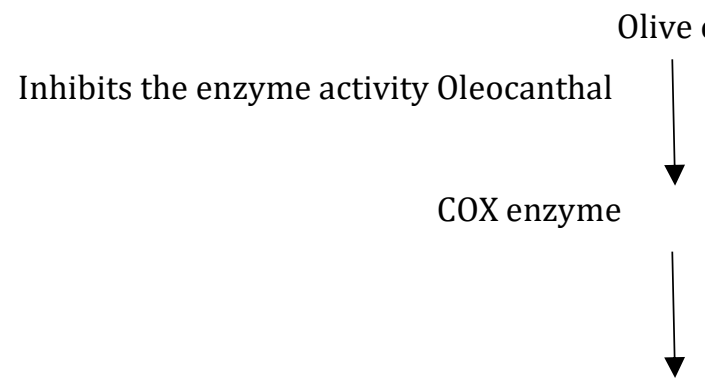

Reduce pain and body inflammatory response (Skoczyńska and Świerkot, 2018).

\section{Functional foods and effects on diabetes}

Diabetes is a chronic and long-term illness with several consequences. Hyperglycemia owing to decreased insulin production, insulin resistance, and beta cell dysfunction define this metabolic disease (Thies et al., 2003). In the management of diabetes, the medical nutrition therapy is play very crucial role. Including, estimation of nutrients and energy intake, counting glycemic load and glycemic index of carbohydrates product, daily protein, fats intake calculation. Along with MNT, the use of functional foods and nutraceutical compounds become the part of MNT as the provide the beyond adequate nutritional benefits. Table 3 Functional foods are scientifically proven the potential health benefits including the improve insulin function, reduce oxidative stress and inflammatory markers in body. The use of functional foods in diabetes help in preventing the development of long-term diabetes related complications such as retinopathy, nephropathy, and CVDs. Numerous functional foods perform diverse mechanism of actions that helps in improve and managing the diabetes health status (Tsai et al., 2017).

\section{Whole grains}

Cereals and grains are the basic source of energy nd carbohydrates in diet. Whole grains consider to be as a functional food for diabetes due to their soluble and insoluble fiber, resistance starches, $\beta$-glucan and have wide range of carotenoids, phenols, phytoestrogens, and phenolic acids. attributed to these bioactive compounds of whole grains-based products helpful against the many metabolic disorders such as diabetes (Singh et al., 2005). Fructooligosccharides, inulin, b-glucan, and resistance starches present in the whole grain-based products that involve on the improving the overall health of gut microbiota. Healthy gut microbiota leads towards the better GIT function, improve the satiety and appetite by controlling the ghrelin production, improve insulin function, decrease inflammation and great impact on lipid level in body (Ueeda et al., 2008). Long term consumption of whole grains, cereals, fiber, germ have great impact against the disease related mortality and morbidity (Savica et al., 2010).

\section{Fruits and vegetables}

Fruits and vegetables are high in dietary fibre, phytochemicals, and vitamins, all of which play an important role in health promotion and illness prevention (Savica et al., 2010). Table 4: Biologically rich phytochemicals fruits and vegetables commodities associated with physiological health benefits in the impediment and managing of diabetes. According to studies, eating fruits and vegetables daily has a significant influence on HbA1c, lipid levels, glycemic management, inflammatory indicators, antioxidant defense, and the risk of diabetes complications (98). 
Table 3. Bioactive composites and functional properties of promising fruits and vegetables.

\begin{tabular}{|c|c|c|}
\hline Fruits/ vegetables & Bioactive compound & Functional property \\
\hline Tomato & $\begin{array}{l}\text { Anthocyanin, lycopene, beta } \\
\text { carotene }\end{array}$ & $\begin{array}{l}\text { Prevent from diabetes induce lipid disorder } \\
\text { Induce antioxidant activity } \\
\text { Reduce the hyperglycemia }\end{array}$ \\
\hline Garlic and onions & $\begin{array}{l}\text { Flavonoids, quercetin, allyl sulfors, } \\
\text { anthocyanins }\end{array}$ & $\begin{array}{l}\text { Reduce the hyperglycemia } \\
\text { Improve insulin function } \\
\text { Improve carbohydrates metabolism } \\
\text { Regulate glycolysis and gluconeogenesis }\end{array}$ \\
\hline Pomegranate & $\begin{array}{l}\text { Anthocyanins, tannins, catechins, } \\
\text { gallic acid, oleanolic acid, ursolic } \\
\text { acid, }\end{array}$ & $\begin{array}{l}\text { Hyperglycemia should be reduced, } \\
\text { Lower your cholesterol and atherogenic risk factors. } \\
\text { Reduce serum resisting levels and improve insulin } \\
\text { resistance caused by obesity, }\end{array}$ \\
\hline Citrus fruits & $\begin{array}{l}\text { Lutein, xanthine, } \beta \text {-carotene, } \\
\text { phytosterols }\end{array}$ & $\begin{array}{l}\text { Reduce the effects of oxidative stress } \\
\text { Improve dyslipidemia by restoring dietary lipid } \\
\text { digestion. } \\
\text { decrease the production of pro-inflammatory } \\
\text { cytokines } \\
\text { decrease lipid peroxidation }\end{array}$ \\
\hline Figs & $\begin{array}{l}\text { catechins, anthocyanins Dietary } \\
\text { fiber, chlorogenic acid, pectin, } \\
\text { flavonoids, gallic acid, }\end{array}$ & $\begin{array}{l}\text { Improve lipid and lipoprotein metabolism } \\
\text { Improve insulin sensitivity }\end{array}$ \\
\hline
\end{tabular}

Table 4. Action of some favorable phytochemical rich functional foods. (Savica et al., 2010)

\begin{tabular}{cl}
\hline \multirow{3}{*}{ Green tea } & Improve antioxidant defense system \\
& Improve glycemic control \\
& Reduce inflammation \\
& Improve glycogen content \\
& Improve insulin sensitivity \\
Olive oil & Reduce the LDL oxidation \\
& Regulate cholesterol metabolism \\
& Reduce the pro inflammatory cytokines \\
& Improve insulin sensitivity \\
Cinnamon & Improve glucose uptake \\
& Antioxidant and anti-inflammatory properties \\
& reduce inflammation, oxidative stress \\
Turmeric & improve carbohydrates and lipid metabolism \\
& prevent diabetic cataract \\
& Improve insulin resistance \\
Fish and seafood & Reduce inflammation \\
& Reduce oxidative stress \\
& Improve metabolic syndrome \\
& Improve insulin sensitivity, improve gut micro biota health, regulate satiety \\
Dairy products and probiotics & Improve oxidative stress level \\
& Low the HbA1c \\
\hline
\end{tabular}

\section{Nuts}

Nuts include almonds, walnuts, pistachios basically wellknown as functional foods due to the presence of many bioactive compounds such as bioactive peptides, high biological proteins, functional fatty acids. Polyphenols, antioxidant vitamins and minerals, anti-inflammatory compounds including magnesium, ellagic acid and $\alpha$ - linolenic acid. Regular consumption of nuts has the potential effect on low-grade inflammation and interleukin 6. In diabetic patient the consumption of nuts is favorably influence by provide the healthy endothelial function and antioxidant and polyphenols from nuts also contribution in diabetes management (Ueeda et al., 2008). 


\section{CONCLUSION}

Metabolic diseases disorders and immune system compromised diseases become the leading cause behind the mortality and morbidity rate over all the world so functional foods and nutraceuticals in metabolic and noncommunicable Diseases presents strategies for the prevention of non-communicable diseases and under nutrition through the use of functional foods and nutraceuticals. Good nutrition, lifestyle modifications and use of functional foods in daily dietary patterns enhanced the nutritional and therapeutic effect of diet. Functional Research has shown that the use of certain functional foods and nutraceuticals, including spices, herbs, and millets, animal foods and plant foods can play a role in the treatment and prevention of various diseases and in health promotion. foods are highly nutritious and promote the potential health benefits along with nutrition. They prevent from nutritional deficiencies, protect against diseases, and promote wellness.

\section{CONFLICT OF INTEREST}

The authors declare that they have no conflicts of interest.

\section{REFERENCES}

Abete, I., D. Parra. A.B. Crujeiras, E. Goyenechea and J.A. Martin. 2008. Specific Insulin Sensitivity and Leptin Responses to a Nutritional Treatment of Obesity via a Combination of Energy Restriction and Fatty Fish Intake. Journal of Human Nutrition and Dietetics, 21(6): 591-600.

Adam, O., C. Beringer. T. Kless, C. Lemmen, A. Adam, M. Wiseman, P. Adam, R. Klimmek and W. Forth. 2003. Anti-Inflammatory Effects of a Low Arachidonic Acid Diet and Fish Oil in Patients with Rheumatoid Arthritis. Rheumatology International, 23(1): 2736.

Adefegha, S.A. 2018. Functional Foods and Nutraceuticals as Dietary Intervention in Chronic Diseases; Novel Perspectives for Health Promotion and Disease Prevention. Journal of Dietary Supplements, 15(6): 977-1009.

Aguirre, R. and J.M. May. 2008. Inflammation in the Vascular Bed; Importance of Vitamin C. Pharmacology and Therapeutics, 119(1): 96103.

Alipour, B., A. Homayouni-Rad, E. Vaghef-Mehrabany, S.K. Sharif, L. Vaghef-Mehrabany, M. AsghariJafarabadi, M.R. Nakhjavani and J. Mohtadi-Nia.
2014. Effects of Lactobacillus Casei Supplementation on Disease Activity and Inflammatory Cytokines in Rheumatoid Arthritis Patients; A Randomized Double-Blind Clinical Trial. International Journal of Rheumatic Diseases, 17(5): 519-527.

Ameye, L.G. and W.S. Chee. 2006. Osteoarthritis and Nutrition. From Nutraceuticals to Functional Foods: A Systematic Review of the Scientific Evidence. Arthritis Research and Therapy, 8(4): 122.

Anderson, G.H. and S.E. Moore. 2004. Dietary Proteins in the Regulation of Food Intake and Body Weight in Humans. The Journal of Nutrition, 134, 974S-979S.

Andrea, D., 2021. Foods Helps to Reduce Arthritis Pain, Health Essentials. The Journal of Nutrition, 125,675S-679S.

Ankola, D.D., B. Viswanad, V. Bhardwaj, P. Ramarao and M. R. Kumar. 2007. Development of Potent Oral Nanoparticulate Formulation of Coenzyme Q10 for Treatment of Hypertension: Can the Simple Nutritional Supplements be used as First Line Therapeutic Agents for Prophylaxis/Therapy? European Journal of Pharmaceutics and Biopharmaceutics, 67(2): 361369.

Asher, M.I., S. Montefort, B. Björkstén, C.K. Lai, D.P. Strachan, S.K. Weiland, H. Williams and ISAAC Phase Three Study Group. 2006. Worldwide Time Trends in the Prevalence of Symptoms of Asthma, Allergic Rhinoconjunctivitis, and Eczema in Childhood: ISAAC Phases One and Three Repeat Multicountry Cross-Sectional Surveys. The Lancet, 368(9537): 733-743.

Block, G., C.D. Jensen, T.B. Dalvi, E.P. Norkus, M. Hudes, P.B. Crawford, N. Holland, E.B. Fung., L. Schumacher and P. Harmatz. 2009. Vitamin C Treatment Reduces Elevated C-Reactive Protein. Free Radical Biology and Medicine, 46(1): 70-77.

Boldo, A., P. Campbell, P. Luthra and W.B. White. 2010. Should the Concentration of Vitamin D Be Measured in all Patients with Hypertension? Journal of Clinical Hypertension (Greenwich, Conn.), 12(3): 149.

Bolton-Smith, C., M. Woodward and H. Tunstall-Pedoe. 1992. The Scottish Heart Health Study. Dietary Intake by Food Frequency Questionnaire and Odds Ratios for Coronary Heart Disease Risk. II. The 
Antioxidant Vitamins and Fibre. European Journal of Clinical Nutrition, 46(2): 85-93.

Cencic, A. and W. Chingwaru. 2010. The Role of Functional Foods, Nutraceuticals, and Food Supplements in Intestinal Health. Nutrients, 2(6): 611-625.

Chaturvedi, P. 2012. Antidiabetic Potentials of Momordica Charantia: Multiple Mechanisms behind the Effects. Journal of Medicinal Food, 15(2): 101-107.

Cortés, B., I. Núñez., M. Cofán, R. Gilabert, A. Pérez-Heras, E. Casals, R. Deulofeu and E. Ros. 2006. Acute Effects of High-Fat Meals Enriched with Walnuts or Olive Oil on Postprandial Endothelial Function. Journal of the American College of Cardiology, 48(8): 1666-1671.

Engelhard, Y.N., B. Gazer and E. Paran. 2006. Natural Antioxidants from Tomato Extract Reduce Blood Pressure in Patients with Grade-1 Hypertension: A Double-Blind, Placebo-Controlled Pilot Study. American Heart Journal, 151(1): 100-E6.

Fraser, G.E. 1999. Nut Consumption, Lipids, and Risk of a Coronary Event. Clinical Cardiology, 22(S3): 11-15.

Fritsche, K. 2006. Fatty Acids as Modulators of the Immune Response. Annual Review of Clinical Nutrition., 26: 45-73.

Gila-Diaz, A., G.H. Carrillo, P. Singh and D. Ramiro-Cortijo. 2021. Specialized Pro-Resolving Lipid Mediators in Neonatal Cardiovascular Physiology and Diseases. Antioxidants, 10(6): 933.

Gu, Y., S. Yu. and J.D. Lambert. 2014. Dietary Cocoa Ameliorates Obesity-Related Inflammation in High Fat-Fed Mice. European Journal of Nutrition, 53(1): 149-158.

Guilleminault, L., E.J. Williams, H.A. Scott, B.S. Berthon, M. Jensen and L.G. Wood. 2017. Diet and Asthma: is it time to adapt our message? Nutrients, 9(11): 1227.

He, J., M.J. Klag, P.K. Whelton, J.Y. Chen, M.C. Qian and G.Q. He. 1995. "Dietary Macronutrients and Blood Pressure in Southwestern China," Journal of Hypertension, 13 (11):1267-1274.

Heber, D. 2004. Vegetables, Fruits and Phytoestrogens in the Prevention of Diseases. Journal of Postgraduate Medicine, 50: 145-149.

Ho, M.J., A. Bellusci and J.M. Wright. 2009. Blood Pressure Lowering Efficacy of Coenzyme Q10 for Primary Hypertension Cochrane Database Systematic Reviews 4:CD007435.

Houston, M. 2013. Nutrition and Nutraceutical Supplements for the Treatment of Hypertension:
Part 1. Alternative and complementary Therapies, 15:752-757.

Houston, M. 2014. The Role of Nutrition and Nutraceutical Supplements in the Treatment of Hypertension. World Journal of Cardiology, 6:3866.

Hu, F.B. and M.J. Stampfer. 1999. Nut Consumption and Risk of Coronary Heart Disease: A Review of Epidemiologic Evidence. Current Atherosclerosis Reports,1(3):204-209.

Huang, Y.D., N. Li and W.G. Zhang. 2011. The Effect of Oral Alpha-Lipoic Acid in Overweight/Obese Individuals on the Brachial-Ankle Pulse Wave Velocity and Supine Blood Pressure: A Randomized, Crossover, Double-Blind, PlaceboControlled Trial. Zhonghua liu xing bing xue za zhi= Zhonghua liuxingbingxue zazhi, 32(3): 290-296.

Jacob, K., M.J. Periago, V. Böhm and G.R. Berruezo. 2008. Influence of Lycopene and Vitamin $\mathrm{C}$ from Tomato Juice on Biomarkers of Oxidative Stress and Inflammation. British Journal of Nutrition, 99(1):137-146.

Jenkins, D.J., C.W. Kendall, C.J.C. Jackson, P.W. Connelly, T. Parker, D. Faulkner, E. Vidgen, S.C. Cunnane, L.A. Leiter and R.G. Josse. 2002. Effects of High-And Low-Isoflavone Soy foods on Blood Lipids, Oxidized LDL, Homocysteine, and Blood Pressure in Hyperlipidemic Men and Women. The American Journal of Clinical Nutrition, 76(2): 365-372.

Kafeshani, M., M.H. Entezari, J. Karimian, M. Pourmasoumi, M.R. Maracy, M.R. Amini and Hadi Aarya. 2017. Atherosclerosis 13(3): 109-116.

Keller, U. 2011. Dietary Proteins in Obesity and Diabetes. International Journal of Vitamins and Nutrition Research, 81(2): 125-133

Khan, M.I., F.M. Anjum, M. Sohaib and A. Sameen.2013. Tackling Metabolic Syndrome by Functional Foods. Reviews in Endocrine and Metabolic Disorders, (3): 287-97.

Korantzopoulos, P., T.M. Kolettis, E. Kountouris, V. Dimitroula, P. Karanikis, E. Pappa, K. Siogas and Goudevenos. 2005. Oral Vitamin C Administration Reduces Early Recurrence Rates After Electrical Cardioversion of Persistent Atrial Fibrillation and Attenuates Associated Inflammation. International Journal of Cardiology, 102(2): 321-326.

Lee, K.P., J.E. Kim, H. Kim, H.R. Chang, D.W. Lee and W.H. Park. 2016. Bo-Gan-Whan regulates proliferation 
and migration of vascular smooth muscle cells. BMC Complementary and Alternative Medicine, 16(1): 1-8.

Lekaki, J., L.S. Rallidis, I. Andreadou, G. Vamvakou, G. Kazantzoglou, P. Magiatis, A.L. Skaltsounis and D.T. Kremastinos. 2005. Polyphenolic Compounds from Red Grapes Acutely Improve Endothelial Function in Patients with Coronary Heart Disease. European Journal of Cardiovascular Prevention Rehabilitation, 12: 596-600.

Lind, L., A. Hänni, H. Lithell, A. Hvarfner, O.H. Sörensen and S. Ljunghall. 1995. Vitamin D is Related to Blood Pressure and Other Cardiovascular Risk Factors in Middle-Aged Men. American Journal of Hypertension, 8(9): 894-901.

Liu, S., J.E. Manson, I.M. Lee, S.R. Cole, C.H. Hennekens, W.C. Willett and J.E. Buring. 2000. Fruit and Vegetable Intake and Risk of Cardiovascular Disease: The Women's Health Study. The American Journal of Clinical Nutrition, 72: 922-928.

Matsunaga, S., K. Azuma, M. Watanabe, T. Tsuka, T. Imagawa, T. Osaki and Y. Okamoto. 2014. Onion Peel Tea Ameliorates Obesity and Affects Blood Parameters in A Mouse Model of High-Fat-DietInduced Obesity. Experimental and Therapeutic Medicine, 7, 379-382.

Medjakovic, S. and A. Jungbauer. 2013. Pomegranate: A Fruit That Ameliorates Metabolic Syndrome. Food and Function, 4, 19-39.

Mikhin, V.P., A.V. Kharchenko, E.A. Rosliakova and M.A. Cherniatina. 2011. Application of Coenzyme Q (10) in Combination Therapy of Arterial Hypertension. Kardiologiia, 51:26-31.

Miller, R.L. and S.M. Ho. 2008. Environmental Epigenetics and Asthma: Current Concepts and Call for Studies. American Journal of Respiratory and Critical Care Medicine, 177(6): 567-573.

Mirmiran, P., Z. Bahadoran and F. Azizi. 2014. Functional Foods-Based Diet as a Novel Dietary Approach for Management of Type 2 Diabetes and Its Complications: A Review. World Journal of Diabetes, 5(3):267-281.

Morcos, M., V. Borcea and B. Isermann. 2001. Effect of Alpha-Lipoic Acid on the Progression of Endothelial Cell Damage and Albuminuria in Patients with Diabetes Mellitus: An Exploratory Study. Diabetes Research and Clinical Practice, 52: 175-183.
Mozaffarian, D. 2008. Fish and N-3 Fatty Acids for the Prevention of Fatal Coronary Heart Disease and Sudden Cardiac Death. The American Journal of Clinical Nutrition 87: 1991S-1996S.

Nestel, P., P. Clifton, D. Colquhoun, M. Noakes, T.A. Mori, D. Sullivan and B. Thomas. 2015.. Indications for omega-3 long chain polyunsaturated fatty acid in the prevention and treatment of cardiovascular disease. Heart, Lung and Circulation, 24(8): 769779.

Ogden, C.L., M.D. Carroll, B.K. Kit and K.M. Flegal. 2014. Prevalence of Childhood and Adult Obesity in the United States, 2011-2012. JAMA, 311: 806-814

Okarter, N. and R.H. Liu. 2010. Health Benefits of Whole Grain Phytochemicals. Critical Reviews in Food Science Nutrition. 50: 193-208.

Paran, E., C. Novac, Y.N. Engelhard and I. Hazan-Halevy. 2009. The Effects of Natural Antioxidants Form Tomato Extract in Treated but Uncontrolled Hypertensive Patients. Cardiovascular Drugs and Therapy, 23:145-151.

Perez-Herrera, A., J. Delgado-Lista, L. Torres-Sanchez, 0. Rangel-Zuñiga, A. Camargo, J. Moreno-Navarrete and C. Muñozlopez. 2012. The Postprandial Inflammatory Response after Ingestion of Heated Oils in Obese Persons is Reduced by the Presence of Phenol Compounds. Molecular Nutrition and Food Research, 56, 510-514.

Prior, R.L., S.E. Wilkes, T.R. Rogers, R.C. Khanal, X. Wu and L.R. Howard. 2010. Purified Blueberry Anthocyanins and Blueberry Juice Alter Development of Obesity in Mice Fed an Obesogenic High Fat Diet. Journal of agriculture and food Chemistry, 58(7): 3970-3976.

Puglisi, M.J., U. Vaishnav, S. Shrestha, M. Torres-Gonzalez, R.J. Wood, J.S. Volek and M.L. Fernandez. 2008. Raisins and Additional Walking have Distinct Effects on Plasma Lipids and Inflammatory Cytokines. Lipids in Health and Disease, 7:14.

Rahman, S.T., N. Merchant, T. Haque, J. Wahi, S. Bhaheetharan, K.C. Ferdinand and B.V. Khan. 2012. The impact of lipoic acid on endothelial function and proteinuria in quinapril-treated diabetic patients with stage I hypertension: results from the QUALITY study. Journal of Cardiovascular Pharmacology and Therapeutics, 17(2): 139-145.

Rayalam, S., M.A. Della-Fera and C.A. Baile. 2008. Phytochemicals and Regulation of the Adipocyte 
Life Cycle. The Journal of Nutritional Biochemistry, 19 (11): 717-726

Reid, K., O.R. Frank and N.P. Stocks. 2009. Dark Chocolate or Tomato Extract for Prehypertension: A Randomized Controlled Trial. BMC Complement Alternative Medicine, 9(1): 1-12.

Riegsecker, S., D. Wiczynski, M.J. Kaplan and S. Ahmed. 2013. Potential Benefits of Green Tea Polyphenol EGCG in the Prevention and Treatment of Vascular Inflammation in Rheumatoid Arthritis. Life Science, 93 (8): 307-12.

Rissanen, T.H., S. Voutilainen, J.K. Virtanen, B. Venho, M. Vanharanta, J. Mursu and J.T. Salonen. 2003. Low Intake of Fruits, Berries and Vegetables is Associated with Excess Mortality in Men: The Kuopio Ischaemic Heart Disease Risk Factor (KIHD) Study. The Journal of Nutrition, 133(1):199-204.

Ros, E., I. Núñez, A. Pérez-Heras, M. Serra, R. Gilabert, E. Casals and R. Deulofeu. 2004. A Walnut Diet Improves Endothelial Function in Hypercholesterolemic Subjects: A Randomized Crossover Trial. Circulation, 1099(13):16091614.

Sackesen, C., H. Ercan, E. Dizdar, O. Soyer, P. Gumus, B.N. Tosun, Z. Buyuktuncer, E. Karabulut, T. Besler and O. Kalayci. 2008. Comprehensive Evaluation of the Enzymatic and Nonenzymatic Antioxidant Systems in Childhood Asthma. Journal of Allergy and Clinical Immunology,122(1): 78-85.

Salinthone, S., R.V. Schillace, C.T. Sang, J.W. Regan, D.N. Bourdette and D.W. Carr. 2011. Lipoic Acid Stimulates cAMP Production Via G Protein-Coupled Receptor-Dependent and -Independent Mechanisms. The Journal of Nutrition and Biochemistry, 22 (7): 681-690.

Savica, V., G. Bellinghieri and J.D. Kopple. 2010. The Effect of Nutrition on Blood Pressure. Annual Review of Nutrition, 30: 365-401.

Scribner, K.B., D.B. Pawlak, C.M. Aubin, J.A. Majzoub and D.S. Ludwig. 2008. Long-Term Effects of Dietary
Glycemic Index on Adiposity, Energy Metabolism, and Physical Activity in Mice. American Journal of Physiology endocrinology and Metabolism, 295 (5), E1126-E1131.

Shaikh, S.R. and M. Edidin. 2008. Polyunsaturated Fatty Acids and Membrane Organization: Elucidating Mechanisms to Balance Immunotherapy and Susceptibility to Infection. Chemistry Physics Lipids,153(1): 24-33a

Singh, U., S. Devaraj and I. Jialal. 2005. Vitamin E, Oxidative Stress, and Inflammation. Annual Review of Nutrition., 25: 151-174.

Skoczyńska. M. and J. Świerkot. 2018. The Role of Diet in Rheumatoid Arthritis. Reumatologia. 56(4): 259267. Doi:10.5114/Reum.2018.77979

Thies, F., J.M. Garry, P. Yaqoob, K. Rerkasem, J. Williams, C.P. Shearman, P.J. Gallagher, P.C. Calder and R.F. Grimble. 2003. Association of N-3 Polyunsaturated Fatty Acids with Stability of Atherosclerotic Plaques: A Randomised Controlled Trial. Lancet 361(9356): 477-485.

Tsai, K.L., C.L. Kao, C.H. Hung, Y.H. Cheng, H.C. Lin and P.M. Chu. 2017. Chicoric Acid is a Potent AntiAtherosclerotic Ingredient by Anti-Oxidant Action and Anti-Inflammation Capacity. Oncotarget, 8(18): 29600.

Ueeda, M., T. Doumei, Y. Takaya, R. Shinohata, Y. Katayama, N. Ohnishi, A. Takaishi, T. Miyoshi, S. Hirohata and S. Kusachi. 2008. Serum N-3 Polyunsaturated Fatty Acid Levels Correlate with the Extent of Coronary Plaques and Calcifications in Patients with Acute Myocardial Infarction. Circulation Journal, 72 (11): 18361843.

Wiseman, H., J.D. O'Reilly, H. Adlercreutz, A.I. Mallet, E.A. Bowey, I.R. Rowland and T.A. Sanders. 2000. Isoflavone Phytoestrogens Consumed in Soy Decrease F2-Isoprostane Concentrations and Increase Resistance of Low-Density Lipoprotein to Oxidation in Humans. The American Journal of Clinical Nutrition, 72(2): 395-400. 
Publisher's note: EScience Press remains neutral with regard to jurisdictional claims in published maps and institutional affiliations.

(c) (†)

Open Access This article is licensed under a Creative Commons Attribution 4.0 International License, which permits use, sharing, adaptation, distribution and reproduction in any medium or format, as long as you give appropriate credit to the original author(s) and the source, provide a link to the Creative Commons license and indicate if changes were made. The images or other third-party material in this article are included in the article's Creative Commons license, unless indicated otherwise in a credit line to the material. If material is not included in the article's Creative Commons license and your intended use is not permitted by statutory regulation or exceeds the permitted use, you will need to obtain permission directly from the copyright holder. To view a copy of this license, visit http://creativecommons.org/licenses/by/4.0/. 